\title{
Current diet does not relate to bone mineral density after the menopause
}

\author{
BY STEVEN A. EARNSHAW, ANN WORLEY AND DAVID J. HOSKING FOR THE \\ NOTTINGHAM EARLY POSTMENOPAUSAL INTERVENTION COHORT \\ (EPIC) STUDY GROUP
}

City Hospital NHS Trust, Hucknall Road, Nottingham NG5 IPB

(Received 9 September 1996 - Revised 25 November 1996 - Accepted 6 January 1997)

\begin{abstract}
The influence of dietary $\mathrm{Ca}$ on peak bone mass and on subsequent bone loss is controversial. Despite this an assessment of nutritional status is often included in the clinical evaluation of osteoporosis risk. To assess the value of this we investigated the relationship between current diet and bone mineral density (BMD) in 426 postmenopausal women, aged 45-59 years, who were enrolled into an international multi-centre trial of alendronate for the prevention of postmenopausal osteoporosis. BMD of the lumbar spine and proximal femur was measured on two occasions approximately 2 weeks apart by dual-energy $X$-ray absorptiometry. Serum osteocalcin was measured by immunoradiometric assay and serum 25 -hydroxycholecalciferol by radioimmunoassay. Dietary assessment was performed by analysis of a $3 \mathrm{~d}$ unweighed dietary record, using Salford University's Microdiet software. BMD at both the lumbar spine and femoral neck correlated significantly with BMI, age, and average serum osteocalcin concentration. We therefore corrected for these variables in subsequent analyses. Dietary Ca intake ranged from 223 to $2197 \mathrm{mg} / \mathrm{d}$ (median $852 \mathrm{mg} / \mathrm{d}$ ). Neither dietary $\mathrm{Ca}$ intake nor any other nutritional variable correlated significantly with BMD. There was a weak, but significant correlation between $\mathrm{Ca}$ intake and serum osteocalcin. We conclude that current diet does not correlate with BMD in early postmenopausal women. However, present diet may affect the rate of change of $\mathrm{BMD}$, and this is supported by the finding of a significant relationship between dietary $\mathrm{Ca}$ and serum osteocalcin, a marker of bone formation rate.
\end{abstract}

Osteoporosis: Calcium: Bone mineral density

The role of nutritional factors in the aetiology of postmenopausal osteoporosis is an area of considerable debate. The importance of dietary $\mathrm{Ca}$ intake and other nutritional factors for skeletal growth during adolescence and in the attainment of peak bone mass is well described, and increased $\mathrm{Ca}$ intake during adolescence has been shown both to increase skeletal growth (Johnston et al. 1992), and to be associated with higher peak bone mass (Ramsdale et al. 1994; Nieves et al. 1995). A retrospective assessment of Ca intake during adolescence has been shown to correlate with bone mineral density (BMD) after the menopause (Murphy et al. 1994).

The relationship between diet and bone mass in early postmenopausal women remains controversial. Epidemiological studies have variously reported either a weak (Elders $e t$ al. 1989; Murphy et al. 1994) or no (Stevenson et al. 1989) relationship between diet and bone mass. Osteoporosis prevention trials using $\mathrm{Ca}$ supplements have either shown only a modest effect (Elders et al. 1991; Reid et al. 1993) or no effect (Dawson-Hughes et al. 1990) in women near the menopause. Despite this conflicting evidence, some form of dietary assessment is often included in the clinical assessment of osteoporosis risk in 
postmenopausal women, and such women are often advised that dietary modifications will reduce their risk of osteoporosis.

The aim of the present study was to investigate whether current diet related to BMD and bone turnover rate in a cohort of healthy early postmenopausal women.

\section{SUBJECTS AND METHODS}

\section{Subjects}

We studied 426 postmenopausal women (mainly in early menopause) who were recruited in Nottingham for inclusion into an international multi-centre, double-blind, placebocontrolled trial of oral alendronate for the prevention of postmenopausal osteoporosis. Women were recruited using population-based techniques from general practitioners' patient lists between September 1992 and June 1993.

Potential subjects were screened by telephone or personal interview, and were considered eligible for the study if they met the following criteria: aged from 45 to 59 years at the time of screening; at least 6 months postmenopausal, confirmed by serum follicle stimulating hormone; of good general health, as judged by medical history, physical examination, and laboratory screening tests; free from lumbar spinal deformity which might prevent precise densitometry; no past or present history of malignancy; no history of disease or treatment affecting bone metabolism (e.g. active thyroid disease, anticonvulsants, corticosteroids); no oestrogen or progestin treatment within 3 months of screening, and no prior bisphosphonate or fluoride therapy; within $\pm 30 \%$ of ideal body weight. Enrolment was restricted so that no more than $10 \%$ of the subjects had a lumbar spine BMD of less than $0.8 \mathrm{~g} / \mathrm{cm}^{2}$ in order to ensure that the majority of the participants were not osteoporotic.

\section{Methods}

All subjects attended two baseline clinic visits, approximately 2 weeks apart before the initiation of therapy. At the first of these a nurse obtained a detailed past medical, menstrual, obstetric, and social history. At the second visit a doctor performed a full clinical history and physical examination.

Bone mineral densitometry was performed at each baseline visit by dual energy X-ray absorptiometry using a Hologic QDR2000 densitometer (Hologic Inc., Waltham, MA, USA). Measurements were made of the lumbar spine and proximal femur. All machine operators attended training sessions conducted by Hologic Inc. Quality assurance was centrally controlled by Hologic Inc., and included daily monitoring of machine performance.

Serum samples were obtained at each baseline visit from all subjects. Serum osteocalcin (reference range $2.4-10 \mathrm{ng} / \mathrm{ml}$ ) was measured by immunoradiometric assay (Nichols Institute Diagnostics Ltd, Saffron Walden, Essex), and 25-hydroxycholecalciferol (reference range $5-72 \mathrm{ng} / \mathrm{ml}$ ) by radioimmunoassay (Incstar Corp., Stillwater, MN, USA). The means of the results from the two baseline samples were used for statistical analysis.

Dietary assessment was performed by a detailed, unweighed, $3 \mathrm{~d}$ dietary record. Subjects kept a record of the type and quantity of all food and drink consumed over a $3 \mathrm{~d}$ period. In order to ensure compliance a descriptive record of portion size rather than a formal weighed record was requested. Weights of food were estimated using a standard reference guide (Crawley, 1988) and analysed by a single trained operator using Microdiet software (Salford University). All analyses were repeated by a second dietitian, who was 
blinded to the first assessment. The nutrient totals were expressed as the mean daily nutrient intakes. Fourteen of the subjects did not return a completed dietary record, and analysis was therefore restricted to the remaining 412 women.

Statistical analysis was performed using Statistical Package for the Social Sciences (SPSS) for Windows ${ }^{\circledR}$ version 6.1 software (1995; SPSS Inc., Chicago, IL, USA). The mean daily nutrient intakes and $95 \% \mathrm{CI}$ for the means were calculated. Multiple linear regression was used to examine the relationship between nutrient intakes, BMI, and BMD. Partial correlation coefficients were calculated for the relationships between $\mathrm{Ca}$ and BMD at the lumbar spine and femoral neck, and between $\mathrm{Ca}$ and osteocalcin.

\section{RESULTS}

The subjects' ages ranged from 45 to 59 years with a median value of 53 years; the duration of menopause ranged from 6 months to 26.5 years with a median of 5.3 years. Ninety-eight (23\%) subjects were smokers, and $206(48 \%)$ had smoked at sometime, but smoking status was not found to relate to either BMD or biochemical markers of bone turnover rate. Alcohol intake, previous hormonal treatment, and previous gynaecological surgery were similarly found to be unrelated to bone mass in this population, and were not therefore considered in further analyses.

The distributions of the subjects' BMI, BMD, average serum osteocalcin, and serum 25-hydroxycholecalciferol results are shown in Table 1.

Reanalysis of the dietary records led to variations of less than $10 \%$ for all nutrients in every subject. The mean daily nutrient intakes (and $95 \% \mathrm{CI}$ for the means) for the study population are presented in Table 2 together with the published UK national average daily nutrient intakes for 1993 (Ministry of Agriculture, Fisheries and Food, 1994) for comparison.

The distribution of dietary $\mathrm{Ca}$ intakes is shown in Fig. 1. Dietary $\mathrm{Ca}$ intake ranged from 223 to $2197 \mathrm{mg} / \mathrm{d}$ with a median intake of $852 \mathrm{mg}$. This is similar to the published 1993 UK national average of $830 \mathrm{mg}$ (Ministry of Agriculture, Fisheries and Food, 1994). A total of $279(68 \%)$ subjects had a dietary Ca intake less than $1000 \mathrm{mg} / \mathrm{d}$, and only twenty $(5 \%)$ subjects had intakes of $1500 \mathrm{mg}$ or higher. The mean dietary intake of vitamin D was $3.05 \mu \mathrm{g}$ (not significantly different from the national average of $2.80 \mu \mathrm{g}$ ).

When the relationships between the various nutrient intakes were examined, that of Ca was found to correlate closely with both phosphate $(r 0.82, P<0.001)$ and lactose ( $r 0.80, P<0.001$ ). Dietary intake of vitamin $\mathrm{D}$ however did not correlate with that of $\mathrm{Ca}$ $(P=0 \cdot 26)$.

Table 1. Distributions of BMI, bone mineral density (BMD, expressed as z-scores of the agematched normal range), serum osteocalcin and serum 25-hydroxycholecalciferol concentrations in 426 postmenopausal women

(Mean values with their standard deviations)

\begin{tabular}{lcccc}
\hline \hline & Mean & SD & Minimum & Maximum \\
\hline BMI $\left(\mathrm{kg} / \mathrm{m}^{2}\right)$ & $25 \cdot 6$ & 3.4 & $16 \cdot 6$ & $36 \cdot 5$ \\
BMD L1-LA z-score & $0 \cdot 11$ & 1.17 & $-2 \cdot 38$ & 3.84 \\
BMD femoral neck z-score & -0.24 & 1.06 & -2.96 & 3.06 \\
Serum osteocalcin (ng/ml) & 7.2 & $2 \cdot 1$ & $2 \cdot 6$ & $18 \cdot 7$ \\
25-Hydroxycholecalciferol (ng/ml) & 11.5 & 9.4 & $2 \cdot 2$ & $82 \cdot 8$ \\
\hline
\end{tabular}


Table 2. Daily nutrient intakes from $3 d$ dietary records for 412 postmenopausal women

\begin{tabular}{|c|c|c|c|}
\hline Nutrient & Mean & $95 \% \mathrm{CI}$ & National average* \\
\hline Energy (kJ) & 7375 & $7199-7549$ & 8125 \\
\hline Protein (g) & $75 \cdot 2$ & $73.4-76.9$ & 62.9 \\
\hline Fat: total $(\mathrm{g})$ & 69.4 & $67 \cdot 0-71 \cdot 7$ & $85 \cdot 0$ \\
\hline Fatty acids: saturated (g) & $22 \cdot 9$ & $21 \cdot 9-24 \cdot 0$ & $33 \cdot 5$ \\
\hline Fatty acids: monounsaturated $(\mathrm{g})$ & $18 \cdot 4$ & $17 \cdot 6-19 \cdot 1$ & 31.4 \\
\hline Fatty acids: polyunsaturated (g) & 5.4 & $5 \cdot 2-5 \cdot 7$ & $14-1$ \\
\hline Carbohydrate $(\mathrm{g})$ & 212 & $206-218$ & 238 \\
\hline Calcium (mg) & 884 & $852-916$ & 830 \\
\hline Sodium (mg) & 2373 & $2287-2459$ & 2520 \\
\hline Potassium (mg) & 3019 & $2940-3097$ & 2580 \\
\hline Magnesium (mg) & 278 & $270-287$ & 233 \\
\hline Iron (mg) & 11.9 & $11 \cdot 4-12 \cdot 4$ & $10 \cdot 2$ \\
\hline Zinc $(\mathrm{mg})$ & 9.4 & $9 \cdot 0-9 \cdot 7$ & 7.9 \\
\hline Vitamin A (retinol equivalent) $(\mu \mathrm{g})$ & 1136 & $1006-1267$ & 1120 \\
\hline Thiamin $(\mathrm{mg})$ & 1.34 & $1 \cdot 25-1.43$ & 1.26 \\
\hline Riboflavin (mg) & 1.97 & $1.88-2.06$ & 1.62 \\
\hline Vitamin $\mathrm{B}_{6}(\mathrm{mg})$ & 1.44 & $1.39-1.49$ & $2 \cdot 0$ \\
\hline Vitamin $\mathrm{B}_{12}(\mu \mathrm{g})$ & $4 \cdot 8$ & $4 \cdot 2-5 \cdot 3$ & 4.9 \\
\hline Folate $(\mu \mathrm{g})$ & 192 & $185-199$ & 245 \\
\hline Vitamin C (mg) & 78 & $73-83$ & 56 \\
\hline Vitamin D $(\mu \mathrm{g})$ & 3.05 & $2.59-3.51$ & 2.8 \\
\hline
\end{tabular}

* Average for all age and sex groups (Ministry of Agriculture, Fisheries and Food, 1994).

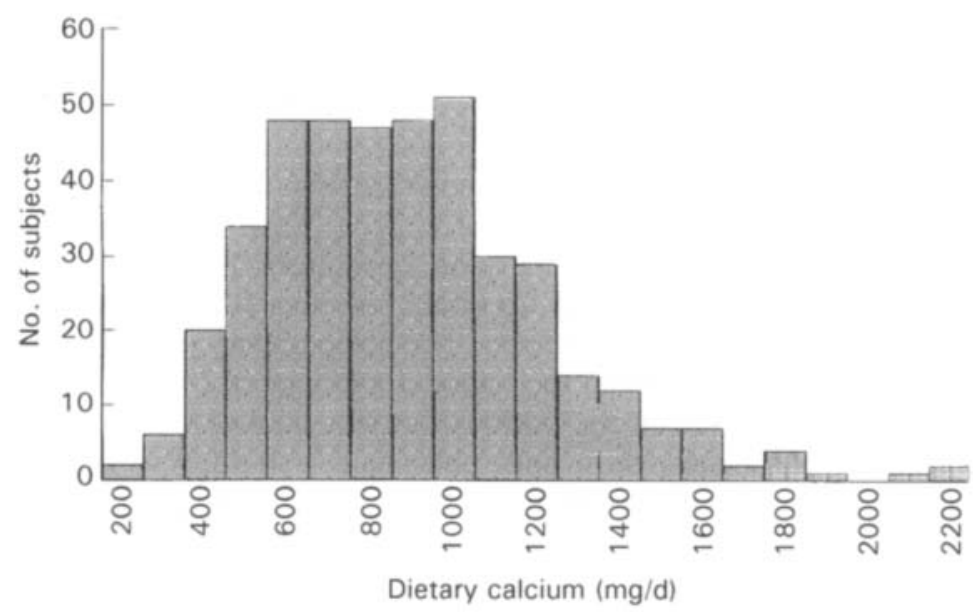

Fig. 1. The distribution of dietary calcium intakes in 412 postmenopausal women from the Early Postmenopausal Intervention Cohort (EPIC) study.

There was no significant correlation between dietary intake of vitamin D and serum 25-hydroxycholecalciferol $(P=0 \cdot 2)$. BMI was weakly related to protein intake $(P=0.0004, r 0.16)$, and to years since the menopause $(P=0.04, r 0.10)$. However BMI was negatively related to carbohydrate $(P=0.04, r-0.10)$, and energy intake $(P=0.03, r-0.10)$, and did not correlate to total dietary fat intake $(P=0.19)$.

Linear multiple regression analysis showed that after age and BMI had been accounted for neither dietary $\mathrm{Ca}$, nor any other dietary factor correlated significantly with $\mathrm{BMD}$ at 
either the lumbar spine or femoral neck. The results of this analysis are shown in Table 3, and the relationships between dietary $\mathrm{Ca}$ and BMD at the lumbar spine and femoral neck are shown in Figs. 2 and 3. The adjusted $r^{2}$ values for these regression equations were 0.05 and 0.12 for the lumbar spine and femoral neck respectively.

There was a significant negative correlation between serum osteocalcin and BMD which persisted after correction for age and BMI $(r-0.17, P<0.001$ for the lumbar spine, and $r-0.19, P<0.001$ for the femoral neck). There was a weak but statistically significant positive correlation between dietary $\mathrm{Ca}$ intake and serum osteocalcin $(r 0.11$, $P=0.028$ ), which persisted after adjustment for BMD and BMI (Fig. 4).

\section{DISCUSSION}

Subjects for this study were recruits for a randomized trial of a new preventative therapy, and our results may therefore have been subject to selection bias. Despite this the mean nutrient intakes of our cohort closely resembled published national averages (Ministry of Agriculture, Fisheries and Food, 1994).

Nutrient intakes assessed from $3 \mathrm{~d}$ unweighed records are subject to both random dayto-day dietary variation and errors of assessment. Day-to-day variations in nutrient intake would lead to errors of between 10 and $20 \%$ for each individual subject (Bingham, 1987), however these errors are random in nature and would be unlikely to mask any clinically relevant relationship in such a large population sample. Because all dietary records were analysed by a single operator errors of assessment should have affected all subjects equally, and are unlikely to have influenced the study results. Furthermore, when records were reassessed by a second dietitian nutrient intakes were not found to differ significantly for any subject. A weighed dietary record might have yielded even more accurate results, however subject compliance may have been somewhat lower. In addition the collection of weighed dietary data in this large population-based study would have required greater resources, and was beyond the scope of this study.

Although the women in this cohort were all aware of the risk of osteoporosis, some $68 \%$ of them had daily intakes of $\mathrm{Ca}$ below the recommended daily allowance for

Table 3. Multiple regression analysis of bone mineral density at the lumbar spine and femoral neck

\begin{tabular}{lrrrr}
\hline & \multicolumn{2}{c}{ Lumber spine } & & \multicolumn{2}{c}{ Femoral neck } \\
\cline { 2 - 3 } \cline { 5 - 5 } Variable & \multicolumn{1}{c}{$\beta$} & \multicolumn{1}{c}{$P$} & & \multicolumn{1}{c}{$\beta$} \\
\hline BMI & 0.204 & $<0.0001$ & 0.326 & $<0.0001$ \\
Age & -0.132 & 0.0069 & -0.174 & 0.0002 \\
Calcium & 0.050 & 0.3089 & 0.077 & 0.1012 \\
Energy & 0.073 & 0.1400 & 0.064 & 0.1814 \\
Magnesium & -0.002 & 0.9739 & 0.185 & 0.6944 \\
Manganese & -0.006 & 0.9007 & -0.042 & 0.3711 \\
Phosphate & -0.050 & 0.2998 & 0.061 & 0.1894 \\
Protein & 0.060 & 0.2163 & 0.048 & 0.2982 \\
Retinol equivalents & -0.051 & 0.2947 & -0.052 & 0.2654 \\
Sodium & -0.006 & 0.9042 & 0.017 & 0.7097 \\
Vitamin D & 0.000 & 0.9945 & -0.048 & 0.3011 \\
YSM & -0.025 & 0.6187 & -0.030 & 0.5361 \\
Zinc & 0.035 & 0.4639 & -0.007 & 0.8812 \\
\hline
\end{tabular}




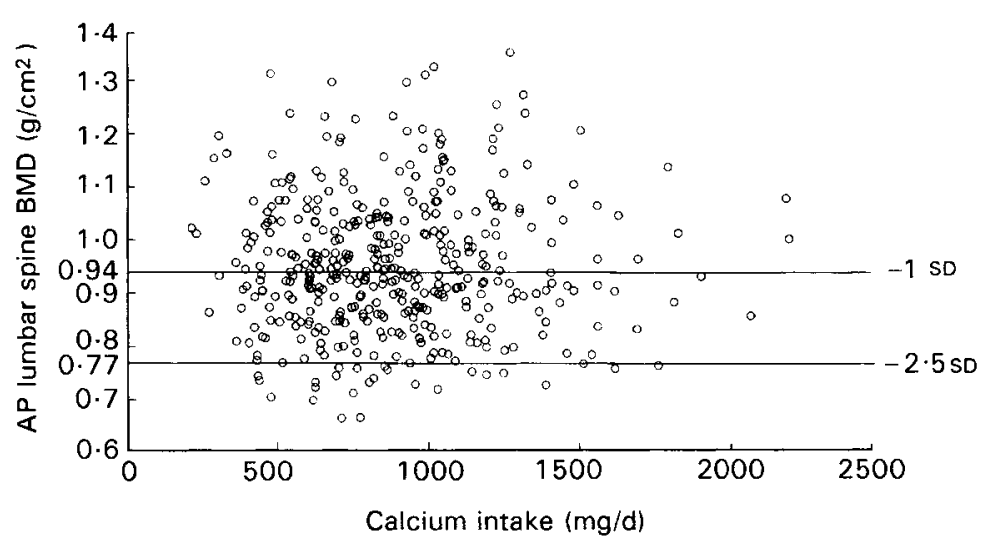

Fig. 2. Dietary calcium intake $v$. anteroposterior (AP) lumber spine bone mineral density (BMD) in 412 postmenopausal women from the Early Postmenopausal Intervention Cohort (EPIC) study. The reference lines indicate the World Health Organization (1994) diagnostic cut-off values for osteopenia ( $-1 \mathrm{SD})$ and osteoporosis $(-2.5 \mathrm{SD})$.

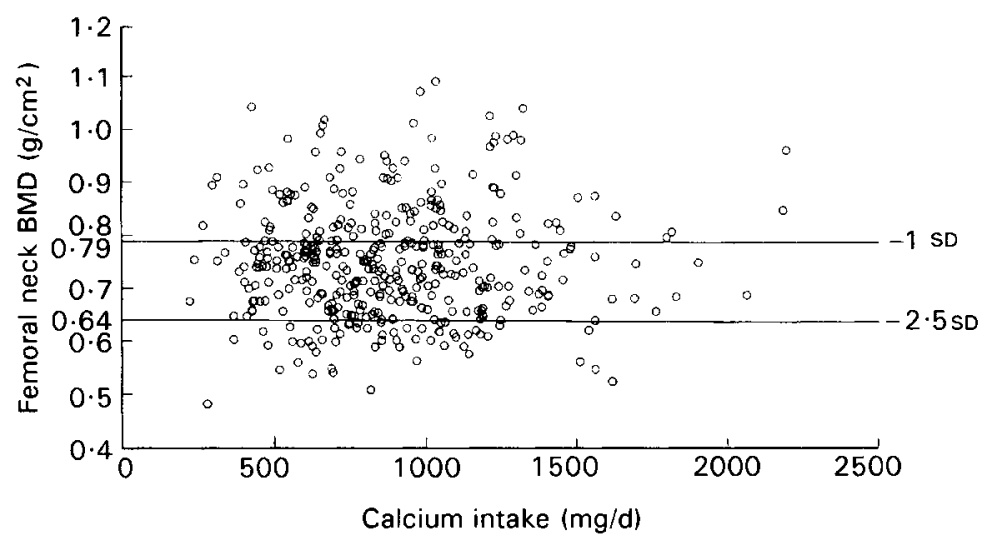

Fig. 3. Dietary calcium intake $v$. femoral neck bone mineral density (BMD) in 412 postmenopausal women from the Early Postmenopausal Intervention Cohort (EPIC) study. The reference lines indicate the World Health Organization (1994) diagnostic cut-off values for osteopenia ( $-1 \mathrm{SD})$ and osteoporosis $(-2.5 \mathrm{SD})$.

postmenopausal women (1000 mg; National Institutes of Health Consensus Development Panel on Optimal Calcium Intake, 1994). It has been suggested that Ca intakes as high as $1500 \mathrm{mg}$ are needed in order to prevent bone loss in oestrogen-deplete postmenopausal women (Heaney, 1993; Selby, 1994), but only $5 \%$ of the present cohort had intakes of this level or higher.

It has been reported previously that dietary phosphate intake has an effect on bone mass independent of $\mathrm{Ca}$ intake (Calvo, 1993). However because $\mathrm{Ca}$ and phosphate intakes were found to be so closely related in this population, separate analysis of phosphate would be unlikely to yield additional useful information.

Many investigators have used either dairy produce or milk consumption as surrogates for total dietary Ca intake (Murphy et al. 1994). Our finding of a close correlation between dietary $\mathrm{Ca}$ and dietary lactose intake (which is derived only from dairy products) supports this assumption. 


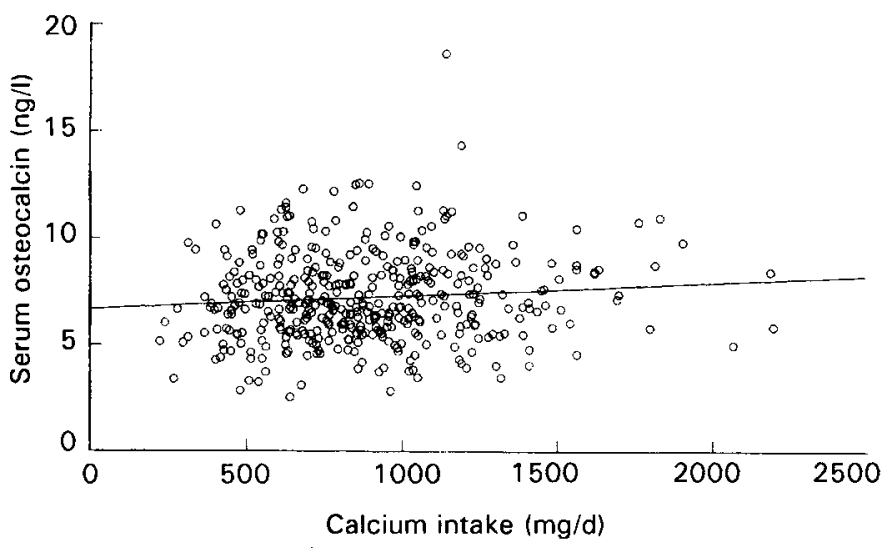

Fig. 4. Dietary calcium $v$. serum osteocalcin in 412 postmenopausal women from the Early Postmenopausal Intervention Cohort (EPIC) study. $r 0.11, P=0.028$.

It is commonly assumed in clinical practice that patients with a good dietary Ca intake will also have adequate vitamin $D$ intake. Our results show, however, that this is not the case. Dietary $\mathrm{Ca}$ and vitamin $\mathrm{D}$ intakes were not related at all, and many subjects with an intake of $\mathrm{Ca}$ above the recommended dietary allowance had very low intakes of vitamin $\mathrm{D}$ and vice versa. In situations where both of these nutrients were thought to be important it would be necessary to measure their intakes separately. We found no relationship between dietary intake of vitamin $\mathrm{D}$ and serum 25 -hydroxycholecalciferol levels. This is consistent with the hypothesis that in active women, who are not housebound, the most important source of vitamin $\mathrm{D}$ is sunlight, and that only when production by the skin is inadequate does dietary intake become of major significance.

Although BMI was found to increase with time from the menopause as expected, it was not related to fat intake and was negatively correlated to carbohydrate and energy intakes. This could be either because the diets of our population have changed over time and do not now resemble those earlier in life when body size was determined, or because more obese subjects underreported intakes of highly energy-dense foods, as has been described previously (Lilienthal Heitmann \& Lissner, 1995).

No dietary factor was found to be related to BMD at either the lumbar spine or proximal femur. This may be because in this population diet has had no effect on bone mass, but this would seem unlikely given the large body of evidence to the contrary. A more likely explanation is that the current diets of this cohort are not representative of those several years ago when their peak bone mass was being attained. This is consistent with the unexpected relationships between BMI and dietary intakes described above. A third possibility is that although diet may have had an influence on skeletal growth and peak bone mass, the subsequent rate of bone loss has been determined entirely by other independent variables, and with increasing time from peak bone mass the relationship between BMD and nutrient intakes has been lost.

If the reason for the absence of any correlation between diet and BMD is that current diets are not the same as those earlier in life, it might still be possible that current diet might determine the current rate of bone loss. Although longitudinal data are not available on this cohort at present, the significant relationship with serum osteocalcin (a marker of bone formation rate) supports this theory. That the correlation to osteocalcin was a positive one does not fit with the findings of treatment trials with $\mathrm{Ca}$ supplements (Elders et al. 
1991; Reid et al. 1993), but a similar positive relationship has been reported between dietary Ca and bone formation rate (Kärkkäinen et al. 1996). This suggests that although at pharmacological doses, Ca suppresses bone turnover, at physiological levels it may have the effect of increasing bone formation rate.

In conclusion, current diet does not relate to BMD in early postmenopausal women. However nutrient intakes may determine the rate of bone loss, and this will be the subject of further study.

We are grateful for the contributions of the many other members of the Nottingham EPIC Research Group, who have made this study possible, and to Merck Sharp \& Dohme Ltd, for funding this research.

\section{REFERENCES}

Bingham, S. (1987). The dietary assessment of individuals; methods, accuracy, new techniques and recommendations. Nutrition Abstracts and Reviews 57, 705-742.

Calvo, M. S. (1993). Dietary phosphorus, calcium metabolism and bone. Journal of Nutrition 123, $1627-1633$. Crawley, H. (1988). Food Portion Sizes. London: H.M. Stationery Office.

Dawson-Hughes, B., Dallal, G. E., Krall, E. A., Sadowski, L., Sahyoun, N. \& Tannenbaum, S. (1990). A controlled trial of the effect of calcium supplementation on bone density in postmenopausal women. New England Journal of Medicine 323, 878-883.

Elders, P. J., Netelenbos, J. C., Lips, P., Khoe, E., van Ginkel, F. C., Hulshof, K. F. A. M. \& van der Stelt, P. F. (1989). Perimenopausal bone mass and risk factors. Bone and Mineral 7, 289-299.

Elders, P. J., Netelenbos, J. C., Lips, P., van Ginkel, F. C., Khoe, E., Leeuwenkamp, O. R., Hackeng, W. H. L. \& van der Stelt, P. F. (1991). Calcium supplementation reduces vertebral bone loss in perimenopausal women: a controlled trial in 248 women between 46 and 55 years of age. Joumal of Clinical Endocrinology and Metabolism 73, 533-540.

Heaney, R. P. (1993). Bone mass, nutrition, and other lifestyle factors. American Journal of Medicine 95, Suppl. 5A, 29-33.

Johnston, C. C., Miller, J. Z., Slemenda, C. W., Reister, T. K., Hui, S., Christian, J. C. \& Peacock, M. (1992). Calcium supplementation and increases in bone mineral density in children. New England Journal of Medicine 327, 82-87.

Kärkkäinen, M., Ray, C., Vanninen, T. \& Lamberg-Allardt, C. (1996). The influence of habitual dietary calcium intake on markers of bone and calcium metabolism. Osteoporosis International 6, Suppl. 1, 152.

Lilienthal Heitmann, B. \& Lissner, L. (1995). Dietary underreporting by obese individuals - is it specific or nonspecific? British Medical Journal 311, 986-989.

Ministry of Agriculture, Fisheries and Food (1994). National Food Survey 1993: Annual Report on Household Food Consumption and Expenditure. London: H.M. Stationery Office.

Murphy, S., Khaw, K.-T., May, H. \& Compston, J. E. (1994). Milk consumption and bone mineral density in middle aged and elderly women. British Medical Journal 308, 939-941.

National Institutes of Health Consensus Development Panel on Optimal Calcium Intake (1994). Optimal calcium intake. Journal of the American Medical Association 272, 1942-1948.

Nieves, J. W., Golden, A. L., Siris, E., Kelsey, J. L. \& Lindsay, R. (1995). Teenage and current calcium intake are related to bone mineral density of the hip and forearm in women aged 30-39 years. American Journal of Epidemiology 141, 342-351.

Ramsdale, S. J., Bassey, E. J. \& Pye, D. J. (1994). Dietary calcium intake relates to bone mineral density in premenopausal women. British Journal of Nutrition 71, 77-84.

Reid, I. R., Ames, R. W., Evans, M. C., Gamble, G. D. \& Sharpe, S. J. (1993). Effect of calcium supplementation on bone loss in postmenopausal women. New England Journal of Medicine 328, $460-464$.

Selby, P. L. (1994). Calcium requirement - a reappraisal of the methods used in its determination and their application to patients with osteoporosis. American Journal of Clinical Nutrition 60, 944-948.

Stevenson, J. C., Lees, B., Devenport, M., Cust, M. P. \& Granger, K. F. (1989). Determinants of bone density in normal women: risk factors for future osteoporosis? British Medical Journal 298, 924-928.

World Health Organization (1994). Assessment of Fracture Risk and its Application to Screening for Postmenopausal Osteoporosis. Report of a WHO Study Group. Geneva: WHO. 